\title{
On the occasion of publication of the feature on diagnosis and assessment of nonalcoholic fatty liver disease/nonalcoholic steatohepatitis using ultrasound elastography
}

\author{
Kazushi Numata ${ }^{1}$ \\ Published online: 3 November 2020 \\ (c) The Japan Society of Ultrasonics in Medicine 2020
}

It is now possible to eliminate the hepatitis $\mathrm{C}$ virus and inhibit proliferation of the hepatitis $\mathrm{B}$ virus, leading to a downward trend in carcinogenesis subsequent to viral hepatitis in Japan, but the occurrence of hepatocellular carcinoma subsequent to non- $\mathrm{B}$, non- $\mathrm{C}$ hepatitis is increasing year by year, with non- $\mathrm{B}$, non- $\mathrm{C}$ hepatitis being the cause in about one-third of hepatic carcinoma cases [1].

The primary origins of non- $\mathrm{B}$, non- $\mathrm{C}$ hepatitis are alcoholic liver disease in individuals with a history of excessive alcohol intake (defined in Japan as $60 \mathrm{~g} /$ day (ethanol equivalent)) and nonalcoholic fatty liver disease (NAFLD) in individuals without a history of alcohol intake or who drink little. NAFLD, which is primarily associated with metabolic syndrome, is a condition in which fatty liver is found on histological examination or diagnostic imaging. The prevalence of NAFLD is increasing as the number of obese and diabetic patients increases. NAFLD is classified as nonalcoholic fatty liver (NAFL), which is considered to be an almost non-progressive entity, and nonalcoholic steatohepatitis (NASH), which is a form of NAFLD with the progression of fibrosis. NAFL and NASH are differentiated by means of liver biopsy, in principle.

According to the Clinical Practice Guideline for NAFLD/ NASH 2020 (revised 2nd edition), NAFLD is defined as follows: (1) Fatty deposition in the liver histologically $\geq 5 \%$ is considered significant. (2) NASH is characterized by steatosis, inflammation, and hepatocellular damage (ballooning degeneration) according to pathological diagnosis. (3) Intertransition of NAFL and NASH is seen, with the progression

Kazushi Numata

kz-numa@urahp.yokohama-cu.ac.jp

1 Gastroenterological Center, Yokohama City University Medical Center, Urafune-cho 4-57, Minami-ku, Yokohama, Kanagawa 232-0024, Japan of fibrosis sometimes occurring despite a slow progression rate in some cases of NAFL. (4) The upper limit of alcohol intake is $30 \mathrm{~g} /$ day for men and $20 \mathrm{~g} /$ day for women (ethanol equivalent). (5) Drug-induced fatty liver disease is fundamentally regarded as drug-induced hepatic injury. (6) Disorders that present so-called microvesicular degeneration such as Reye syndrome and acute fatty liver of pregnancy are excluded from NAFLD. (7) In some cases of NASH liver cirrhosis, the characteristics of NASH such as steatosis and ballooning degeneration disappear as it progresses, resulting in burned-out NASH. Furthermore, the pathological finding most associated with the vital prognosis is liver fibrosis; therefore, the degree of fibrosis should be considered when selecting the method of follow-up observation and treatment.

There are some issues that arise due to the fact that NAFLD/NASH are diagnosed by exclusion. One issue is that the history of alcohol intake itself is self-reported. Another issue is that in the case of fatty liver in individuals who neither drink too much nor drink little alcohol, i.e., men who drink 30-60 g/day and women who drink 20-60 g/day (ethanol equivalent), it does not fall under the category of either alcoholic liver disease or NAFLD/NASH. In any event, the prevalence of chronic liver injury due to metabolic disease has increased in recent years, and their diagnosis and treatment are a matter of urgency.

NAFLD, which is a cause of chronic liver disease, is said to afflict over 200 million people worldwide, and the prevalence in Japan has been reported to be $29.7-32.2 \%$ in studies in individuals who have undergone screening [2, 3].

Liver cirrhosis and carcinogenesis subsequent to NAFLD/ NASH are reflected in the prognosis. Carcinogenesis subsequent to NASH (5.29/1000 person-years) occurs more frequently than carcinogenesis subsequent to NAFLD (0.44/1000 person-years) [4]. Moreover, it has been reported 
that about one or two out of every 100 people with NAFLD will ultimately develop liver cancer [5].

It should be noted that it has been reported that $38 \%$ of patients with carcinogenesis subsequent to NASH did not have coexisting liver cirrhosis. In other words, the risk of developing liver cancer is high even in cases without progression of fibrosis [6].

Thus, NAFLD/NASH are important clinical entities, but unlike conventional viral liver diseases, patients with NAFLD/NASH do not undergo periodic diagnostic imaging, and therefore liver cancer subsequent to NAFLD/NASH is often detected at a more advanced stage than that attributable to hepatitis $\mathrm{C}[7,8]$.

Proper diagnosis of NAFLD/NASH before carcinogenesis and periodic testing thereafter will be critical for early detection. NASH has conventionally been diagnosed histopathologically by means of liver biopsy, but biopsy is an invasive procedure, and although rare, it can cause serious complications such as bleeding. In addition, the size of the liver tissue that can be collected with a biopsy gun with an $18 \mathrm{G}$ needle is about $15 \mathrm{~mm}$ in length, which does not necessarily reflect the state of the entire liver. Moreover, the diagnosis can vary depending on the pathologist. Key noninvasive tests (NITs) that can be performed repeatedly include not only serum markers such as the fibrosis-4 (FIB-4) index and the NAFLD fibrosis score (NFS) but also ultrasound (US) elastography and magnetic resonance (MR) elastography. The American Association for the study of Liver Disease (AASLD) Practice Guide 2018 recommends four NITs to evaluate hepatic fibrosis, including the FIB-4 index, the NFS, vibration-controlled transient elastography (VCTE), and MR elastography [9].

In addition to VCTE, US elastography available on several devices is covered by health insurance in Japan, and the fibrosis markers type IV collagen 7S and Mac-2 binding protein glycosylation isomer (M2BPGi) are also covered by insurance.

Various drugs are currently being developed as therapeutic agents for NASH, and it is hoped that they will eventually be commercially available, but when they do become available, it will be necessary to repeatedly monitor the improvement of fibrosis in NASH over time. For the reasons mentioned above, performing a liver biopsy every time monitoring is necessary is not realistic. It has been reported that MR elastography provides higher precision in the assessment of liver fibrosis than US elastography as a noninvasive examination [10]. The usefulness of MR elastography is not in doubt, but the handier US elastography is easier to use in clinical practice for repeatedly performing examinations over time.

Dr. Toshifumi Tada has provided a comprehensive explanation of NAFLD/NASH that includes not only US elastography but also epidemiology, pathogenesis, fibrosis markers (FIB-4 index, NAFLD fibrosis score), assessment of hepatic steatosis, and more. He has also discussed a proposal to switch from NAFLD to the concept of metabolic dysfunction-associated fatty liver disease (MAFLD) [11]. The proposed criteria for a positive diagnosis of MAFLD are based on histological (biopsy), imaging, or blood biomarker evidence of fat accumulation in the liver (hepatic steatosis), in addition to one of the following three criteria: overweight/ obesity, presence of type 2 diabetes mellitus, or evidence of metabolic dysregulation.

Dr. Masato Yoneda has described the characteristics of US elastography techniques such as Real-time Tissue Elastography ${ }^{\circledR}$ (RTE), VCTE, point shear wave elastography, and two-dimensional shear wave elastography, and has compared them with liver biopsy. In addition, he has explained the limitations and advantages of each US elastography modality in an easy-to-understand manner. He has also explained the assessment of hepatic steatosis that uses controlled attenuation parameters and attenuation imaging.

Dr. Kento Imajo has compared US elastography and MR elastography, in which he explains that MR elastography is expensive and not easy to use, but the entire liver is observable and liver fat accumulation can be assessed using proton density fat fraction measurement and, therefore, assessment of fibrosis and liver fat accumulation is more precise than US elastography. He has also explained the diagnosis of NASH using non-elastographic MR techniques and assessment of portal hypertension using MRI in NAFLD patients.

Dr. Masayoshi Kage has explained histological features of NAFLD on the basis of steatosis, inflammation, ballooning hepatocytes, Mallory-Denk bodies, and fibrosis, and explained pathological differentiation between NAFLD and NASH. Ballooning hepatocytes and Mallory-Denk bodies may be useful pathological findings for diagnosing NASH. The area from the portal triads to the hepatic vein is divided into three regions. Zone 1 encircles the portal tracts, where the oxygenated blood from hepatic arteries enters. Zone 3 is located around central veins, where oxygenation is poor [12, 13]. Zone 3 fibrosis or perivenular fibrosis initially develops in NASH. In contrast, zone 1 fibrosis develops in chronic viral hepatitis. Pericellular fibrosis in zone 3 is a characteristic pattern of NASH as well as alcoholic steatohepatitis.

I believe these four review articles will contribute to a more comprehensive understanding of not only ultrasound elastography but also diagnosis and assessment of NAFLD/ NASH.

In closing, ultrasound attenuation occurs due to subcutaneous fat and fatty liver itself in obese individuals, and therefore it is considered to be unsuitable for the detection of liver cancer as the deep part of the liver cannot be visualized well. Contrast-enhanced CT and contrast-enhanced MRI are generally recommended. On the other hand, severely obese individuals are sometimes too big to fit into 
the contrast-enhanced CT or contrast-enhanced MRI unit. In such cases, US may be the only means of screening for lesions. Overseas medical device manufacturers have developed and marketed probes that enable deep observation with US in severely obese individuals. I would like to see Japanese manufacturers develop ultrasound devices that allow for deep observation in severely obese individuals. I hope that we will be able to diagnosis fibrosis and detect liver cancer using a single US system in the near future.

\section{References}

1. Tateishi R, Uchino K, Fujiwara N, et al. A nationwide survey on non-B, non-C hepatocellular carcinoma in Japan: 2011-2015 update. J Gastroenterol. 2019;54:367-76.

2. Eguchi Y, Hyogo H, Ono M, et al. Prevalence and associated metabolic factors of nonalcoholic fatty liver disease in the general population from 2009 to 2010 in Japan: a multicenter large retrospective study. J Gastroenterol. 2012;47:586-95.

3. Hamaguchi M, Takeda N, Kojima T, et al. Identification of individuals with non-alcoholic fatty liver disease by the diagnostic criteria for the metabolic syndrome. World J Gastroenterol. 2012;7:1508-16.

4. Younossi ZM, Koenig AB, Abdelatif D, et al. Global epidemiology of nonalcoholic fatty liver disease-meta-analytic assessment of prevalence, incidence, and outcomes. Hepatology. 2016;64:73-84.

5. Sumida Y, Yoneda M, Seko Y, et al. Surveillance of hepatocellular carcinoma in nonalcoholic fatty liver disease. Diagnostics (Basel). 2020;10:579
6. Tokushige K, Hashimoto E, Horie Y, Taniai M, Higuchi S. Hepatocellular carcinoma in Japanese patients with nonalcoholic fatty liver disease, alcoholic liver disease, and chronic liver disease of unknown etiology: report of the nationwide survey. J Gastroenterol. 2011;46:1230-7.

7. Piscaglia F, Svegliati-Baroni G, Barchetti A, et al. Clinical patterns of hepatocellular carcinoma in nonalcoholic fatty liver disease: a multicenter prospective study. Hepatology. 2016;63:827-38.

8. Mittal S, Sada YH, El-Serag HB, et al. Temporal trends of nonalcoholic fatty liver disease-related hepatocellular carcinoma in the veteran affairs population. Clin Gastroenterol Hepatol. 2015;13:594-601.

9. Chalasani N, Younossi Z, Lavine JE, et al. The diagnosis and management of nonalcoholic fatty liver disease: practice guidance from the American Association for the study of liver diseases. Hepatology. 2018;67:328-58.

10. Imajo K, Kessoku $\mathrm{T}$, Honda $\mathrm{Y}$, et al. Magnetic resonance imaging more accurately classifies steatosis and fibrosis in patients with nonalcoholic fatty liver disease than transient elastography. Gastroenterology. 2016;150:626-637e7.

11. Eslam M, Newsome PN, Sarin SK, et al. A new definition for metabolic dysfunction-associated fatty liver disease: an international expert consensus statement. J Hepatol. 2020;73:202-9.

12. Jungermann K, Kietzmann T. Zonation of parenchymal and nonparenchymal metabolism in liver. Annu Rev Nutr. 1996;16:179-203.

13. Jungermann K, Kietzmann T. Oxygen: modulator of metabolic zonation and disease of the liver. Hepatology. 2000;31:255-60.

Publisher's Note Springer Nature remains neutral with regard to jurisdictional claims in published maps and institutional affiliations. 